\title{
Noise Characterization of a Multi-Channel Receiver Using a Small Antenna Array with Full Diversity for Robust Satellite Navigation
}

\author{
Safwat Irteza* ${ }^{(1)}$, Eric Schäfer ${ }^{(2)}$, Christian Volmer, Matteo Sgammini( ${ }^{(3)}$, Ralf Stephan ${ }^{(1)}$, \\ Eckhard Hennig $^{(2)}$, Matthias A. Hein ${ }^{(1)}$ \\ (1) RF and Microwave Laboratory, Ilmenau University of Technology, Germany \\ (2) Institut für Mikroelektronik- und Mechatronik-Systeme gemeinnützige GmbH, Germany \\ (3) German Aerospace Center (DLR), Institute for Communication and Navigation, Germany \\ E-mail: safwat-irteza.butt@tu-ilmenau.de
}

\section{Introduction}

We develop a miniaturized multi-antenna L-band receiver for robust global navigation systems (GNSS) in the framework of an industrial-academic publicly funded research project. In contrast to previous successful implementation [1], our goal is to provide the benefits of array processing on a smaller geometrical scale, where compactness is achieved by reducing both the size of the individual antenna elements as well as their inter-element distances. In order to maintain full diversity as required for robust satellite navigation algorithms, we employ a decoupling and matching network DMN as proposed earlier [2]. DMNs are passive circuits; they generate noise proportional to their ohmic losses. Since the DMN forms an integral part of the antenna, it must be connected directly to the feed ports of the array. Therefore, it must be placed in front of the first amplifier, resulting in a noise penalty that might outweigh the intended gain in diversity. So far, DMNs have often been considered lossless [3, 4]. To our knowledge, it has not yet been proven that real, i.e. dissipative, DMNs can be used beneficially for lownoise receivers.

In this paper, the noise contribution of the DMN is accurately taken into account in terms of the equivalent system noise temperature. We provide detailed insight into the noise performance of a small antenna array system, including a network exciting matched eigenmodes, low-noise amplifiers, and digital beamforming. It is shown that the $\mathrm{DMN}$ is necessary to reduce the equivalent noise contribution of the LNAs significantly, especially for full exploitation of pattern diversity. It is observed that noise matching using a DMN results in a reduction of the equivalent system noise temperature by up to a factor of three (or $4.8 \mathrm{~dB}$ ) compared to the conventional version of an otherwise identical array.

\section{Model of the GNSS Diversity Receiver}

According to Fig. 1, the diversity receiver comprises four components: antenna array, DMN, LNAs, and

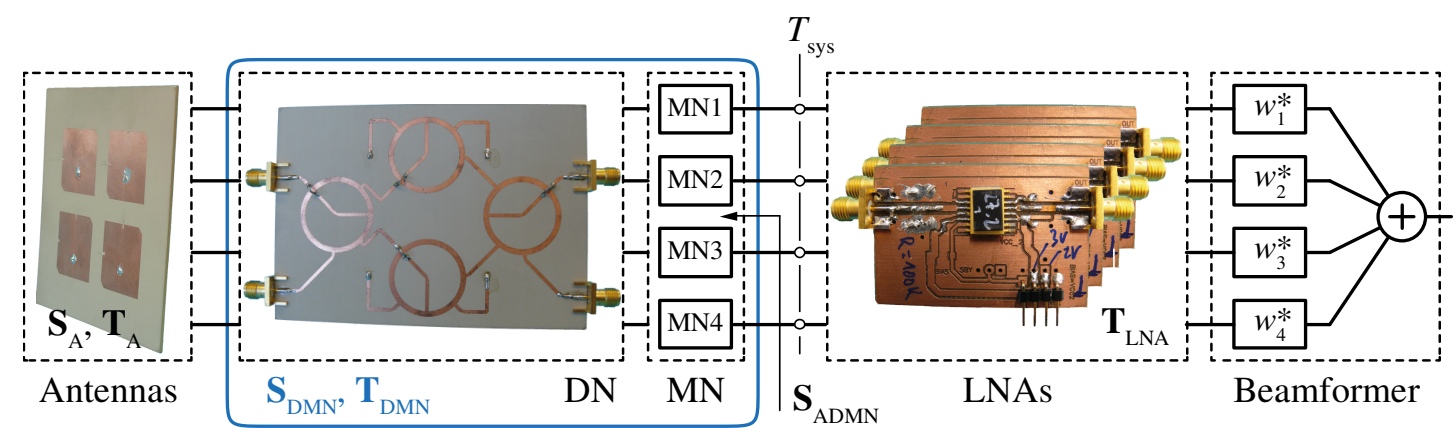

Fig. 1. Receiver model in full-diversity configuration: DN and $\mathrm{MN}$ denote the decoupling network and the matching network, respectively. $\mathbf{S}_{\mathrm{ADMN}}$ indicates the combined S-parameter matrix of antenna array and DMN. The conventional configuration excludes the components inside the blue frame. 
beamformer. The antenna is composed of a $2 \times 2$ array of corner-truncated square patches on RO3010 with a grid spacing of $d=\lambda / 5$. The overall size of the antenna is $10 \mathrm{~cm} \times 10 \mathrm{~cm}$. The measured right-hand circularly polarized (RHCP) radiative eigen-efficiencies are $56 \%, 16 \%, 6 \%$, and $1 \%$ for modes \#1 (even), \#2 (odd1), \#3 (odd2), and \#4 ( $\pi$-mode), respectively.

The DMN is implemented in two blocks. The first stage is composed of four 3-dB $180^{\circ}$ hybrid couplers (Fig. 1). The measured S-parameters of the decoupled antenna array are fed into a lossy matchingnetwork model, designed with Agilent ADS, to achieve either power or noise matching to the LNA, depending on the operational strategy.

Four inductively degenerated common-source cascode stages with lumped LC loads and input tanks were employed for low-noise amplification. They have been implemented in a commercial $0.35-\mu \mathrm{m}$ CMOS process. Each amplifier was mounted to a standard FR4-based printed circuit board. Measurements revealed an input impedance of $Z_{\text {in }}=50 \Omega$, a minimal noise figure of $F_{\min }=1.7 \mathrm{~dB}$, a noise resistance of $R_{\mathrm{n}}=6 \Omega$, and a noise-matching impedance of $Z_{\mathrm{opt}}=(26+\mathrm{j} 0.3) \Omega$.

We consider a typical deterministic beamformer for determining the complex-valued weights for a desired direction of arrival (DoA) across the upper hemisphere. As we focus here exclusively on the noise contribution of the DMN, the spatial scenario is simplified to an interferer-free situation. The diversity capability of the antenna array plays no role in this case.

\section{Receiver Performance Characterization}

We use the carrier-to-receiver noise density ratio at the output of the beamformer as the figure of merit of the receiver. This is a convenient and straightforward approach since it contrasts the unequal influence on the carrier and noise components of the received signal. The carrier-to-receiver noise density ratio $\chi=C / N_{0}$ is a scalar value, with $C$ and $N_{0}$ the equivalent available carrier power and the equivalent available noise power spectral density, respectively, referred to the LNA inputs. $C$ and $N_{0}$ can be expressed in analytical form, incorporating measured far-field patterns, S-parameters, and noise parameters of antenna array, DMN, and amplifiers, respectively.

The equivalent available carrier power is calculated from

$$
C(\phi, \theta)=C_{\mathrm{sat}} \mathbf{w}^{\mathrm{H}} \mathbf{G}_{\mathrm{DMN}} \mathbf{F}(\phi, \theta) \mathbf{F}^{\mathrm{H}}(\phi, \theta) \mathbf{G}_{\mathrm{DMN}}^{\mathrm{H}} \mathbf{w},
$$

where $C_{\text {sat }}$ is the power received with an ideal RHCP isotropic antenna, and $\mathbf{w}$ is the vector of the weighting coefficients in the beamformer. The individual elements of the column vector $\mathbf{F}(\phi, \theta)$ denote the normalized complex-valued realized RHCP amplitude gain of the single antennas with respect to an $*_{-}$ isotropic radiator. $\mathbf{G}_{\mathrm{DMN}}$ is the effective gain matrix of the DMN:

$$
\mathbf{G}_{\mathrm{DMN}}=\mathbf{S}_{\mathrm{DMN}, 12}\left(\mathbf{I}-\mathbf{S}_{\mathrm{A}} \mathbf{S}_{\mathrm{DMN}, 22}\right)^{-1} \text {. }
$$

$\mathbf{S}_{\mathrm{DMN}}$ and $\mathbf{S}_{\mathrm{A}}$ are the S-parameter matrices of the DMN and the antenna array, respectively. $\mathbf{S}_{\mathrm{DMN}, i j}$ is the two-port equivalent $S$-matrix from port $i$ to port $j$. The reference plane 1 refers to the interface between DMN and LNA, while plane 2 refers to the interface between DMN and antenna. The S-parameters of the LNA are irrelevant since they affect the carrier and noise signal components equally. If no DMN is included in the receiver, the gain matrix of the DMN simplifies to $\mathbf{G}_{\mathrm{DMN}}=\mathbf{I}$.

We derive the noise power spectral density from the equivalent system noise temperature $T_{\text {sys }}$, referred to the LNA inputs:

$$
N_{0}=k_{\mathrm{B}} T_{s y s}=k_{\mathrm{B}} \underbrace{\mathbf{w}^{\mathrm{H}} \mathbf{T}_{\mathrm{ADMN}} \mathbf{w}}_{T_{\mathrm{ADMN}}}+k_{\mathrm{B}} \underbrace{\frac{\mathbf{w}^{\mathrm{H}} \mathbf{T}_{\mathrm{LNA}} \mathbf{w}}{\mathbf{w}^{\mathrm{H}}\left(\mathbf{I}-\mathbf{S}_{\mathrm{ADMN}} \mathbf{S}_{\mathrm{ADMN}}^{\mathrm{H}}\right) \mathbf{w}}}_{T_{\mathrm{LNA}}} .
$$


The combined noise temperature correlation matrix of antenna array and DMN is calculated from

$$
\mathbf{T}_{\mathrm{ADMN}}=\mathbf{G}_{\mathrm{DMN}} \mathbf{T}_{\mathrm{A}} \mathbf{G}_{\mathrm{DMN}}^{\mathrm{H}}+\mathbf{T}_{\mathrm{DMN}},
$$

where $\mathbf{T}_{\mathrm{A}}$ and $\mathbf{T}_{\mathrm{DMN}}$ denote the noise temperature correlation matrices of antenna array and DMN, respectively. $\mathbf{T}_{\mathrm{A}}$ includes noise received from the environment as well as from the antenna losses:

$$
\mathbf{T}_{\mathrm{A}}=T_{\text {env }} \mathbf{H}_{\mathrm{A}}^{\mathrm{T}}+T_{\text {amb }}\left(\left(\mathbf{I}-\mathbf{S}_{\mathrm{A}} \mathbf{S}_{\mathrm{A}}^{\mathrm{H}}\right)-\mathbf{H}_{\mathrm{A}}\right)^{\mathrm{T}} .
$$

$T_{\text {env }}=100 \mathrm{~K}$ is the assumed equivalent isotropic environmental temperature for GNSS conditions; $T_{\mathrm{amb}}=290 \mathrm{~K}$ is the ambient temperature of the antennas. $\mathbf{H}_{\mathrm{A}}$ denotes the radiation matrix of the array:

$$
\mathbf{H}_{\mathrm{A}}=\frac{1}{4 \pi} \oiint \mathbf{F}(\phi, \theta) \mathbf{F}^{\mathrm{H}}(\phi, \theta) \mathrm{d} \Omega
$$

$\mathbf{T}_{\text {DMN }}$ can be obtained from the S-parameters of antenna array and DMN:

$$
\mathbf{T}_{\mathrm{DMN}}=\mathbf{T}_{v}+\mathbf{T}_{\rho} \mathbf{S}_{\mathrm{A}}^{\mathrm{H}} \mathbf{G}_{\mathrm{DMN}}^{\mathrm{H}}+\mathbf{G}_{\mathrm{DMN}} \mathbf{S}_{\mathrm{A}} \mathbf{T}_{\rho}^{\mathrm{H}}+\mathbf{G}_{\mathrm{DMN}} \mathbf{S}_{\mathrm{A}} \mathbf{T}_{\mu}^{\mathrm{H}} \mathbf{S}_{\mathrm{A}}^{\mathrm{H}} \mathbf{G}_{\mathrm{DMN}}^{\mathrm{H}}
$$

$\mathbf{T}_{\text {DMN }}$ includes the noise occurring in the DMN as well as noise which is reflected back from the antennas due to mismatch and mutual coupling. The parameters in (7) read:

$$
\begin{aligned}
& \mathbf{T}_{v}=T_{\mathrm{amb}}\left(\mathbf{I}-\mathbf{S}_{\mathrm{DMN}, 11} \mathbf{S}_{\mathrm{DMN}, 11}^{*}-\mathbf{S}_{\mathrm{DMN}, 21}^{\mathrm{T}} \mathbf{S}_{\mathrm{DMN}, 21}^{*}\right), \\
& \mathbf{T}_{\mu}=T_{\mathrm{amb}}\left(\mathbf{I}-\mathbf{S}_{\mathrm{DMN}, 22} \mathbf{S}_{\mathrm{DMN}, 22}^{*}-\mathbf{S}_{\mathrm{DMN}, 21} \mathbf{S}_{\mathrm{DMN}, 21}^{\mathrm{H}}\right), \\
& \mathbf{T}_{\rho}=T_{\mathrm{amb}}\left(-\mathbf{S}_{\mathrm{DMN}, 11} \mathbf{S}_{\mathrm{DMN}, 21}^{\mathrm{H}}-\mathbf{S}_{\mathrm{DMN}, 21}^{\mathrm{T}} \mathbf{S}_{\mathrm{DMN}, 22}^{*}\right) .
\end{aligned}
$$

The contribution due to the amplifiers is calculated according to Warnick et al. [5]. The equivalent noise temperature correlation matrix is defined as:

$$
\mathbf{T}_{\mathrm{LNA}}=\left(\mathbf{T}_{\alpha}+\mathbf{S}_{\mathrm{ADMN}} \mathbf{T}_{\beta} \mathbf{S}_{\mathrm{ADMN}}^{\mathrm{H}}-\mathbf{S}_{\mathrm{ADMN}} \mathbf{T}_{\gamma}-\mathbf{T}_{\gamma}^{\mathrm{H}} \mathbf{S}_{\mathrm{ADMN}}^{\mathrm{H}}\right),
$$

where the S-matrices of array and DMN are combined as:

$$
\mathbf{S}_{\mathrm{ADMN}}=\mathbf{S}_{\mathrm{DMN}, 11}+\mathbf{S}_{\mathrm{DMN}, 21}^{\mathrm{T}} \mathbf{S}_{\mathrm{A}} \mathbf{G}_{\mathrm{DMN}}^{\mathrm{T}} \cdot
$$

We assume that the noise generated by one LNA is uncorrelated with all other amplifiers. Therefore, the input-referred noise correlation matrices in (11) simplify to $\mathbf{T}_{\alpha}=T_{\alpha} \mathbf{I}, \mathbf{T}_{\beta}=T_{\beta} \mathbf{I}$, and $\mathbf{T}_{\gamma}=T_{\gamma} \mathbf{I}$, in which $T_{\alpha}$, $T_{\beta}$, and $T_{\gamma}$ are calculated from the measured noise parameters $F_{\min }, R_{\mathrm{n}}$, and $Z_{\mathrm{opt}}$ [6]. If no DMN is employed in the receiver chain, we have $\mathbf{T}_{\mathrm{DMN}}=\mathbf{0}, \mathbf{S}_{\mathrm{DMN}, 11}=\mathbf{S}_{\mathrm{DMN}, 22}=\mathbf{0}$, and $\mathbf{S}_{\mathrm{DMN}, 12}=\mathbf{S}_{\mathrm{DMN}, 21}=\mathbf{I}$.

\section{Results and Discussion}

To evaluate the system performance, $\chi=C / N_{0}$ has been calculated for Galileo E1-band signals where $C_{\text {sat }}=-157 \mathrm{dBW}$ [7]. In a first step, $\mathbf{w}$ was fixed to the respective eigenvectors, and $\chi$ was calculated for all directions across the upper hemisphere. The resulting noise temperatures with and without DMN are shown in Table I. It can be seen that noise matching reduces the system noise temperature for mode \#4 $(\pi)$ by a factor of three, a truly significant improvement. Similarly, as depicted in Fig. 2, the maximum $\chi$ value is improved by approximately $10 \mathrm{~dB}$ for the $\pi$-mode, which is beneficial for robust satellite navigation in the presence of interferers. However, for the amplifier chosen, there is no clear advantage in terms of matching strategies, since $Z_{\text {opt }} \approx Z_{\text {in }}$. In the next step, the direction of arrival was swept across the upper hemisphere, and the beamforming weights for each direction were used to calculate $\chi$; the results are displayed in Fig. 3. It is evident that a DMN improves the system performance due to an increased matching efficiency.

\section{Conclusions}

The employment of a DMN in small antenna arrays results in an increased antenna noise temperature due to increased ohmic losses. On the other hand, it minimizes the amplifier noise contribution considerably, thus reducing the equivalent system noise temperature. Therefore, the use of a DMN for small antenna 


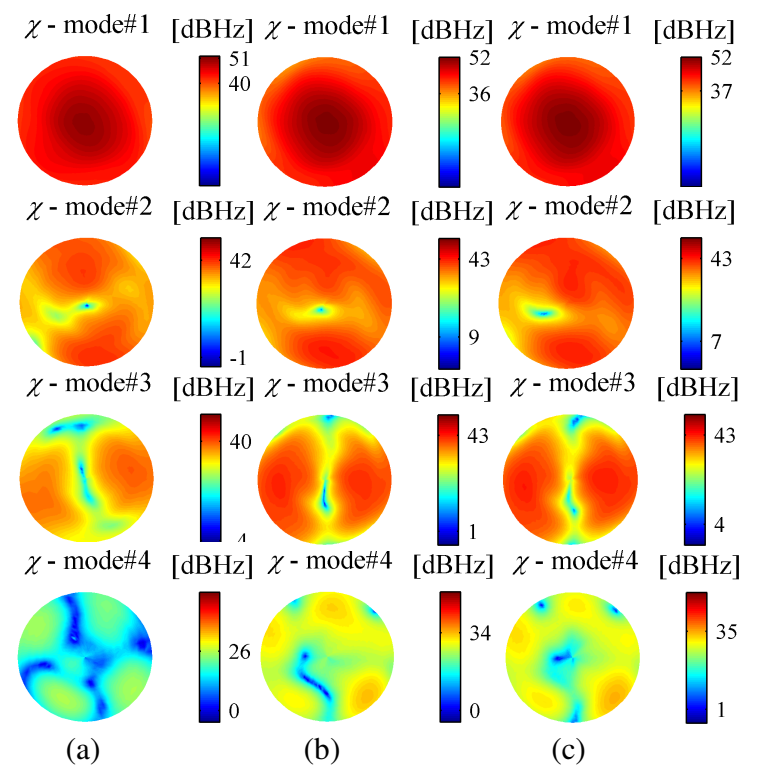

Fig. 2. $C / N_{0}$ for all spatial directions for fixed eigenmode excitation weights, without DMN (a), for power matching (b), and for noise matching (c). Highest and lowest values are given in the legends.
TABLE I

EQUIVALENT NOISE TEMPERATURES

\begin{tabular}{|c|c|c|c|c|c|c|}
\hline \multirow[b]{3}{*}{ Mode } & & & \multicolumn{4}{|c|}{ With DMN } \\
\hline & \multicolumn{2}{|c|}{$\begin{array}{c}\text { Without } \\
\text { DMN }\end{array}$} & \multicolumn{2}{|c|}{$\begin{array}{c}\text { Power } \\
\text { matching }\end{array}$} & \multicolumn{2}{|c|}{$\begin{array}{c}\text { Noise } \\
\text { matching }\end{array}$} \\
\hline & $T_{\mathrm{A}}$ & $T_{\mathrm{LNA}}$ & $T_{\mathrm{ADMN}}$ & $T_{\mathrm{LNA}}$ & $T_{\mathrm{ADMN}}$ & $T_{\mathrm{LNA}}$ \\
\hline$\# 1$ & 156 & 171 & 169 & 156 & 148 & 145 \\
\hline \#2 & 144 & 439 & 242 & 171 & 223 & 151 \\
\hline \#3 & 203 & 216 & 221 & 152 & 200 & 158 \\
\hline \#4 & 74 & 1120 & 276 & 164 & 236 & 155 \\
\hline & & & wer & {$[\mathrm{dBH}$} & no & \\
\hline
\end{tabular}

Fig. 3. $C / N_{0}$ across the upper hemisphere after applying beamforming weights. Highest and lowest values are given in the legends.

arrays displaying full diversity is not only beneficial but necessary for optimizing receiver performance. The analysis sketched here will be extended to null-steering or interferer-cancellation scenarios.

\section{Acknowledgements}

The LNA has been made available by our colleagues A. Richter and B. Bieske. Technical assistance has been provided by M. Huhn and M. Zocher. This work was funded by the Space Administration of the German Aerospace Center (DLR) on behalf of the Federal Ministry of Economics and Technology under grant number 50NA1007.

\section{References}

[1] M. V. T. Heckler, M. Cuntz, A. Konovaltsev, L. A. Greda, and M. Meurer, "Development of Robust Safety-of-Life Navigation Receivers," IEEE Trans. Microw. Theory Tech., vol. 59, no. 4, pp. 998 1005, April 2011.

[2] C. Volmer, J. Weber, R. Stephan, K. Blau, and M. A. Hein, "An Eigen-Analysis of Compact Antenna Arrays and Its Application to Port Decoupling," IEEE Trans. on Ant. and Prop., vol. 56, no. 2, pp. 360-370, Feb. 2008.

[3] K. F. Warnick and M. A. Jensen "Optimal Noise Matching for Mutually Coupled Arrays," IEEE Trans. on Ant. and Prop., vol. 55, no. 6, pp. 1726-1731, Jun. 2007.

[4] M. T. Ivrlač and J. A. Nossek, "On the Diversity of Compact Antenna Arrays," URSI-GASS, Aug. 2011.

[5] K. F. Warnick, et al., "Unified Definitions of Efficiencies and System Noise Temperature for Receiving Antenna Arrays," IEEE Trans. on Ant. and Prop., vol. 58, no. 6, pp. 2121-2125, Jun. 2010.

[6] J. Engberg and T. Larsen, Noise Theory of Linear and Nonlinear Circuits, Chichester: J. Wiley \& Sons, 1995.

[7] (2010, Sep.). Galileo OS SIS ICD. [Online]. Available: http://ec.europa.eu/enterprise/policies/satnav/galileo/files/galileo-os-sis-icd-issue1-revision1_en.pdf 